\title{
New techniques for cost sharing in combinatorial optimization games
}

\author{
Alberto Caprara • Adam N. Letchford
}

Received: 3 June 2008 / Accepted: 10 August 2009 / Published online: 11 May 2010

(C) Springer and Mathematical Programming Society 2010

\begin{abstract}
Combinatorial optimization games form an important subclass of cooperative games. In recent years, increased attention has been given to the issue of finding good cost shares for such games. In this paper, we define a very general class of games, called integer minimization games, which includes the combinatorial optimization games in the literature as special cases. We then present new techniques, based on row and column generation, for computing good cost shares for these games. To illustrate the power of these techniques, we apply them to traveling salesman and vehicle routing games. Our results generalize and unify several results in the literature. The main underlying idea is that suitable valid inequalities for the associated combinatorial optimization problems can be used to derive improved cost shares.
\end{abstract}

Keywords Cooperative games · Optimal cost shares ·

Combinatorial optimization games · Valid inequalities · Traveling salesman game ·

Vehicle routing game

Mathematics Subject Classification (2000) $\quad 91 \mathrm{~A} 12 \cdot 91 \mathrm{~A} 46 \cdot 05 \mathrm{C} 57 \cdot 90 \mathrm{C} 05 \cdot$ $90 \mathrm{C} 10 \cdot 90 \mathrm{C} 27 \cdot 90 \mathrm{C} 57$

\footnotetext{
A. Caprara $(\varangle)$

DEIS, University of Bologna, Viale Risorgimento 2,

40136 Bologna, Italy

e-mail: alberto.caprara@unibo.it

\section{A. N. Letchford}

Department of Management Science, Lancaster University,

Lancaster LA1 4YW, England

e-mail: A.N.Letchford@lancaster.ac.uk
} 


\section{Introduction}

Cooperative game theory is concerned with situations in which, rather than competing, the players are able to cooperate by forming independent coalitions (see, for example, $[28,32,37])$. In this paper, we are concerned with cost sharing games with Transferable Utilities (TU cost sharing games). In such games, a group of players is willing to pay for some kind of service. The cost of serving all players, or any subset of players, is known. The task is to share costs among the players in a 'fair' way, which means that there is no incentive for any subgroup of players to break away from the group and form their own coalition.

More formally, let $V$ be the player set, with $|V|=n$. The set of potential coalitions is denoted by $\mathcal{S}$. (Usually, $\mathcal{S}=2^{V} \backslash\{\emptyset\}$.) For any $S \in \mathcal{S}$, let $c(S)$ denote the cost of serving the players in $S$. (The cost of serving all players is then $c(V)$.) Notice that, for a given coalition $S$, we do not need to know how much each player in $S$ would actually contribute to the cost $c(S)$. In other words, the cost $c(S)$ is 'transferrable' from one player to another within $S$. In non-TU games this is not the case. For more details about non-TU games, see [32].

A cost share is a set of weights $w_{i}$ for $i \in V$ that satisfies the following core property:

$$
\sum_{i \in S} w_{i} \leq c(S), \quad S \in \mathcal{S} .
$$

Ideally, one would like to find a cost share that is budget balanced, i.e., such that:

$$
\sum_{i \in V} w_{i}=c(V)
$$

Unfortunately, such a cost share may not exist.

A key concept in the literature is that of the core [12], which is the set of budget balanced cost shares, viewed as a polyhedron in $\mathbb{R}^{n}$. If no budget balanced cost share exists, the core is empty. To address games with empty core, various cost shares have been defined which are 'good' in various senses, such as the nucleolus, the kernel, the $\varepsilon$-core, and so on (see [32]). An idea of relevance here is that of $\gamma$-budget balanced cost shares, i.e., cost shares that satisfy

$$
\sum_{i \in V} w_{i} \geq \gamma c(V)
$$

for some $\gamma \in[0,1]$. Recently, researchers have devoted some attention to the problem of finding a cost share that is $\gamma$-budget balanced for the maximum possible $\gamma$ (see the survey [23]). This problem, which we call the Optimal Cost Share Problem (OCSP), amounts to solving the following Linear Program (LP):

$$
\max \left\{\sum_{i \in V} w_{i}: \quad \sum_{i \in S} w_{i} \leq c(S), \quad S \in \mathcal{S}\right\} .
$$


There are several motives for wishing to solve the OCSP, either exactly or approximately. First, the framework models the situation where a public institution, such as local government, is subsidising a public service. Given a cost share $w$, the institution can charge player $i$ the quantity $w_{i}$, leaving the quantity $c(V)-\sum_{i \in V} w_{i}$ to be paid by the institution. Then, clearly, the institution has an incentive to find an optimal (or near-optimal) cost share. Second, if a cost share $w$ is $\gamma$-budget balanced, then by charging each player $i$ the quantity $w_{i} / \gamma$ we can ensure that, for any coalition $S \in \mathcal{S}$, we are charging no more than $c(S) / \gamma$. If $\gamma$ is close to 1 , then $w / \gamma$ may be acceptable in practice. Third, there exist natural TU cost sharing games for which the OCSP can be solved efficiently even though testing whether the core is non-empty is $\mathcal{N} \mathcal{P}$-hard. (An example is given in Sect. 5.) Fourth, the dual of Eq. 3 arises naturally in the computation of bounds for cutting, packing, routing, scheduling and clustering problems, and many other combinatorial optimization problems.

Several important TU cost sharing games arising in MS/OR are combinatorial optimization games, in which $c(S)$ can be computed by solving a combinatorial optimization problem. These include, for example, the assignment game [36], the spanning tree game [16], the traveling salesman game [38], the vehicle routing game [15], the packing and covering games [7] and the facility location game [14].

In recent years, researchers have established the existence of approximation algorithms for several TU combinatorial optimization games. That is, they have shown that there is a constant $\gamma>0$ such that one can find a $\gamma$-budget balanced cost share in polynomial time (see, e.g., [2,9,14,23]). Researchers have also examined approximate cost sharing mechanisms for non-TU variants (see the survey [23]).

In this paper, we take a somewhat different approach to the OCSP. We begin by defining a very general class of games, called Integer Minimization (IM) games, which includes as special cases all TU combinatorial optimization games of which we are aware. We then take two highly successful existing techniques for solving combinatorial optimization problems - namely the cutting plane and column generation techniques - and show how to use them to compute 'good' (though not necessarily optimal) cost shares for IM games. We also illustrate the power of these new techniques by applying them to various games. Our results generalize and unify several results in the literature. The main underlying idea is that suitable valid inequalities for the associated combinatorial optimization problems can be used to derive improved cost shares.

The remainder of the paper is structured as follows. In Sect. 2, we formally define the IM games and show how various TU combinatorial optimization games fall in this class. Our new methods for computing good cost shares are presented in Sect. 3. The methods are then illustrated on the covering and facility location games in Sect. 4, on the two-matching and unrooted traveling salesman games in Sect. 5, and on the traveling salesman and vehicle routing games in Sect. 6. Finally, we give some concluding remarks in Sect. 7.

We remark that the methods we propose in this paper can easily be adapted to the 'dual' case in which revenues rather than costs have to be shared among players, and we wish to obtain a revenue share which is as small as possible. For brevity, however, we concentrate on the case of costs. 


\section{Integer minimization games}

As mentioned in the introduction, the results in this paper apply to a class of games that we call IM games. In this section, we define the IM games and show how various combinatorial optimization games fall in this class.

\subsection{Definition and basic properties}

In order to define the IM games, we need the following notation. Recall that $\mathcal{S} \subseteq$ $2^{V} \backslash\{\emptyset\}$ denotes the family of potential coalitions (where $V \in \mathcal{S}$ ). Although traditionally $\mathcal{S}=2^{V} \backslash\{\emptyset\}$, we permit some coalitions to be forbidden, as explained below. Furthermore, for any $S \subseteq V, y(S)$ denotes the incidence vector of $S$, i.e., the vector in $\{0,1\}^{n}$ defined by $y_{i}(S)=1$ if $i \in S, y_{i}(S)=0$ otherwise. The integer minimization games are then defined as follows.

Definition 1 A game with player set $V$ and coalition family $\mathcal{S}$ is called an Integer Minimization (IM) game if there exist:

- positive integers $p$ and $m$,

- a matrix $A \in \mathbb{Z}^{p \times m}$,

- a matrix $B \in \mathbb{Z}^{p \times n}$,

- a right hand side vector $d \in \mathbb{Z}^{p}$,

- an objective function vector $c \in \mathbb{Z}^{m}$,

such that, for all $S \in \mathcal{S}$, the $\operatorname{cost} c(S)$ is equal to the solution value of the following Integer Linear Program (ILP):

$$
c(S)=\min \left\{c x: A x \geq B y(S)+d, x \in \mathbb{Z}_{+}^{m}\right\} .
$$

We assume that ILP (Eq. 4) is feasible and bounded for all $S \in \mathcal{S}$.

Note that $c(V)=\min \left\{c x: A x \geq B \mathbf{1}+d, x \in \mathbb{Z}_{+}^{m}\right\}$, where $\mathbf{1}$ denotes the all-ones vector of dimension $n$. Note also that every combinatorial optimization game can be formulated as an IM game by letting $m:=|\mathcal{S}|$, i.e., by introducing one variable for every coalition. (This is analogous to the fact that every combinatorial optimization problem can be formulated as an ILP by introducing one variable for each feasible solution.) Of course, in cases of practical interest, $m$ is much smaller, typically polynomial in $|V|$.

A class of games analogous to IM games was defined in [13], who considered the games for which $c(S)$ is given by the optimal solution of an LP in which the feasible region depends on $S$ but the objective function does not.

To close this subsection, we present two general results concerned with IM games.

Observation 1 Every IM game with $d$ non-negative is subadditive; that is, satisfies $c(S \cup T) \leq c(S)+c(T)$ for all $S, T \in \mathcal{S}$ such that $S \cup T \in \mathcal{S}$ and $S \cap T=\emptyset$.

Proof Consider $S, T \in \mathcal{S}$ such that $S \cup T \in \mathcal{S}$ and $S \cap T=\emptyset$, letting $x^{S}$ and $x^{T}$ be optimal solutions of ILP (Eq. 4) for $S$ and $T$, respectively. It is easy to verify that $x^{S}+x^{T}$ is a feasible solution of Eq. 4 for $S \cup T$, implying $c(S \cup T) \leq c\left(x^{S}+x^{T}\right)=c(S)+c(T)$. 
Observation 2 Every IM game with $B$ non-negative is monotonic; that is, satisfies $c(S \cup\{i\}) \geq c(S)$ for all $S \in \mathcal{S}$ and $i \in V \backslash S$ such that $S \cup\{i\} \in \mathcal{S}$.

Proof Consider $S \in \mathcal{S}$ and $i \in V \backslash S$ such that $S \cup\{i\} \in \mathcal{S}$, letting $x^{S \cup\{i\}}$ be an optimal solution of ILP (Eq. 4) for $S \cup\{i\}$. It is easy to check that the non-negativity of $B$ implies that $x^{S \cup\{i\}}$ is a feasible solution of Eq. 4 for $S$, implying $c(S) \leq c(S \cup\{i\})$.

Many of the IM games we consider in this paper satisfy $d=0$. We call such IM games homogeneous. Note that homogenous IM games are subadditive, but need not be monotonic.

\subsection{Some specific IM games}

We now show that several specific TU combinatorial optimization games in the literature can be easily formulated as IM games. We refer the reader to the references in which these games are introduced for their interpretation in terms of players and strategies.

Packing and covering games are examined in [7]. Their covering games are nothing but the IM games in which $\mathcal{S}=2^{V} \backslash\{\emptyset\}, B$ is the identity matrix, $A$ is binary and $d=0$. As shown in [7], many well-known games on graphs fall into this special case.

The so-called facility location games are considered in [14,31]. In our terminology, these are games in which $\mathcal{S}=2^{V} \backslash\{\emptyset\}, d=0$, and $c(S)$ has the following form:

$$
\begin{gathered}
c(S)=\min \left\{\sum_{j=1}^{q} f_{j} v_{j}+\sum_{i \in V} \sum_{j=1}^{q} c_{i j} u_{i j}: \sum_{j=1}^{q} u_{i j}=y_{i}(S), \quad i \in V,\right. \\
\left.\left(v_{j}, u_{1 j}, \ldots, u_{n j}\right) \in F_{j}, \quad j=1, \ldots, q\right\},
\end{gathered}
$$

where, for $j=1, \ldots, q, F_{j}=\{(0, \ldots, 0)\} \cup\left\{(1, y(R)): R \in \mathcal{R}_{j}\right\}$, with $\mathcal{R}_{j} \subseteq$ $2^{V} \backslash\{\emptyset\}$ and $y(R)$ incidence vector of set $R \in \mathcal{R}_{j}$. Here, $\{1, \ldots, q\}$ represents the set of potential facilities and $\mathcal{R}_{j}$ the family of the player subsets that can be served by facility $j$. Note that Eq. 5 is not an ILP. On the other hand, such an ILP, leading to an IM game formulation, can easily be obtained from the specific structure of $\mathcal{R}_{j}$ for the cases of practical interest. For instance, in the basic uncapacitated case, in which $\mathcal{R}_{j}=2^{V} \backslash\{\emptyset\}$ for $j=1, \ldots, q$, the constraint $\left(v_{j}, u_{1 j}, \ldots, u_{n j}\right) \in F_{j}$ can be expressed by $u_{i j} \leq v_{j} \leq 1, u_{i j}, v_{j} \in \mathbb{Z}_{+}$for $i \in V$.

Next, we consider the traveling salesman game, considered for example in $[9,29$, $33,38]$. In this game, one is given a complete undirected graph $G=(V \cup\{0\}, E)$, with edge $\operatorname{costs} c_{e} \geq 0$ for all $e \in E$. The vertex 0 represents a depot. For a given set $S$ of players, $c(S)$ is the minimum cost of a Hamiltonian circuit on $S \cup\{0\}$, i.e., of a circuit passing through $S \cup\{0\}$ and not passing through any vertex in $V \backslash S$. This game can be formulated as an IM game with $\mathcal{S}=2^{V} \backslash\{\emptyset\}$. Indeed, for any $S \in \mathcal{S}$, the following holds: 


$$
\begin{gathered}
c(S)=\min \left\{c x: \sum_{e \in \delta(0)} x_{e}=2,\right. \\
\quad \sum_{e \in \delta(i)} x_{e}=2 y_{i}(S), \quad i \in V, \\
\sum_{e \in \delta(R)} x_{e} \geq 2 y_{i}(S), \quad R \subseteq V, i \in R, \\
\left.x \in \mathbb{Z}_{+}^{|E|}\right\} .
\end{gathered}
$$

Here, as usual in the literature, $\delta(R)$ denotes the set of edges with one endpoint in $R$ and the other in $V \backslash R$, and we write $\delta(i)$ rather than $\delta(\{i\})$ for brevity.

The vehicle routing game, studied in [15], is similar to the traveling salesman game. However, we are also given a vehicle capacity $Q>0$ and, for each $i \in V$, a demand $q_{i}>0$. For a given set $S$ of players, $c(S)$ is the minimum cost of a set of circuits, each of which visits the depot vertex 0 and a set of players whose total demand does not exceed $Q$, such that each player in $S$ is visited by exactly one circuit (and no player in $V \backslash S$ is visited). This game can be formulated as an IM game with $\mathcal{S}=2^{V} \backslash\{\emptyset\}$ and $d=0$ because, for any $S \in \mathcal{S}$, the following holds:

$$
\begin{gathered}
c(S)=\min \left\{c x: \sum_{e \in \delta(i)} x_{e}=2 y_{i}(S), \quad i \in V,\right. \\
\quad \sum_{e \in \delta(R)} x_{e} \geq 2 \frac{\sum_{i \in R} q_{i} y_{i}(S)}{Q}, \quad R \subseteq V, \\
\left.x \in \mathbb{Z}_{+}^{|E|}\right\} .
\end{gathered}
$$

In [10], a variant of the traveling salesman game is considered in which there is no depot, and $c(S)$ is simply the minimum cost of a Hamiltonian circuit on $S$. For this game, coalitions with $|S|<3$ are forbidden, i.e., $\mathcal{S}=\{S \subseteq V:|S| \geq 3\}$. This is because this game is used here to introduce the analysis of the two more complex games above: by allowing coalitions with $|S|=2$ the game would have a too simple structure. In [10], this variant is called the traveling preacher game, but here we will call it the unrooted traveling salesman game, to emphasize the connection with the standard traveling salesman game. When $|S| \geq 3$, we have:

$$
\begin{aligned}
& c(S)=\min \left\{c x: \sum_{e \in \delta(i)} x_{e}=2 y_{i}(S), \quad i \in V,\right. \\
& \sum_{e \in \delta(R)} x_{e} \geq 2\left(y_{i}(S)+y_{j}(S)-1\right), \quad R \subseteq V, i \in R, j \in V \backslash R, \\
& x_{(i, j)} \leq y_{i}(S), \quad(i, j) \in E, \\
& \left.x \in \mathbb{Z}_{+}^{|E|}\right\} \text {. }
\end{aligned}
$$


In order to derive results for the unrooted traveling salesman game, it will prove useful to consider the following closely related game, which is new to the best of our knowledge and which we call the two-matching game. We have a complete undirected graph $G=(V, E)$, with edge $\operatorname{costs} c_{e}$ for all $e \in E$. Coalitions with $|S|<3$ are again forbidden, whereas for coalitions with $|S| \geq 3, c(S)$ is the minimum cost of a set of circuits visiting all vertices of $S$. That is,

$$
\begin{gathered}
c(S)=\min \left\{\begin{array}{ll}
c x: & \sum_{e \in \delta(i)} x_{e}=2 y_{i}(S), \quad i \in V, \\
& x_{(i, j)} \leq y_{i}(S), \quad(i, j) \in E, \\
& x \in \mathbb{Z}_{+}^{|E|}
\end{array}\right\} .
\end{gathered}
$$

Note that the covering, facility location, vehicle routing and two-matching games have been formulated as homogeneous IM games, and are therefore subadditive. (The covering and facility location games are also monotonic.) On the other hand, it is possible to show that neither of the two traveling salesman games can be formulated as a homogeneous IM game. Indeed, the traveling salesman games are neither monotonic nor subadditive in general, and therefore (by Observation 1) cannot be formulated as IM games in which $d \geq 0$.

However, we can consider the metric versions of the traveling salesman games, arising when the edge or arc costs satisfy the triangle inequality, i.e., $c_{(i, k)} \geq c_{(i, j)}+c_{(j, k)}$ for each triple of distinct vertices $i, j, k$ of $G$. These metric games are both subadditive and monotonic. Indeed, it is not hard to see that, for the metric version of all routing games considered, $c\left(S_{1}\right)+c\left(S_{2}\right) \geq c\left(S_{1} \cup S_{2}\right)$ for all sets $S_{1}, S_{2} \in \mathcal{S}$.

\section{Three methods for computing good allocations}

Given that the OCSP is $\mathcal{N} \mathcal{P}$-hard for many important games, we are led to consider algorithms for computing 'good', though not necessarily optimal, cost shares. In this section, we explain how cost shares can be computed using column generation, row generation, or both. In each case, we show that there is a strong connection with methods for computing lower bounds for $c(V)$ itself, i.e., with lower bounds for

$$
\min \left\{c x: A x \geq B \mathbf{1}+d, x \in \mathbb{Z}_{+}^{m}\right\}
$$

We will need the following concepts. The dual of the LP (Eq. 3) is:

$$
\min \left\{\sum_{S \in \mathcal{S}} c(S) z_{S}: \sum_{S \ni i} z_{S}=1, \quad i \in V, \quad z_{S} \geq 0, \quad S \in \mathcal{S}\right\} .
$$

It can be interpreted as the LP relaxation of a set partitioning problem in which players correspond to constraints and coalitions to variables. The classical Bondareva-Shapley 
theorem $[3,35]$ states that the core is non-empty if and only if the optimal solution value of Eq. 11 is at least $c(V)$.

We use the concepts of convex hulls and conic hulls in what follows. The convex hull of a set of vectors $v_{1}, \ldots, v_{r} \in \mathbb{R}^{m}$, denoted by $\operatorname{conv}\left\{v_{1}, \ldots, v_{r}\right\}$, is given by:

$\operatorname{conv}\left\{v_{1}, \ldots, v_{r}\right\}:=\left\{x \in \mathbb{R}^{m}: x=\sum_{i=1}^{r} \lambda_{i} v_{i}\right.$ for some $\lambda \in \mathbb{R}_{+}^{r}$ such that $\left.\sum_{i=1}^{r} \lambda_{i}=1\right\}$.

The conic hull, denoted by cone $\left\{v_{1}, \ldots, v_{r}\right\}$, is given by:

$$
\operatorname{cone}\left\{v_{1}, \ldots, v_{r}\right\}:=\left\{x \in \mathbb{R}^{m}: x=\sum_{i=1}^{r} \lambda_{i} v_{i} \text { for some } \lambda \in \mathbb{R}_{+}^{r}\right\} \text {. }
$$

\subsection{Column generation}

The first method, based on column generation, is derived in a straightforward way. Note that $c(V)$ is given by the solution of the following ILP:

$$
c(V):=\min \left\{c x: A x \geq B y+d, y=\mathbf{1}, x \in \mathbb{Z}_{+}^{m}\right\} .
$$

We will apply Dantzig-Wolfe decomposition [5] to this ILP, keeping the equations $y=\mathbf{1}$ in the master. To this end, we denote by $Q^{x y}$ the overall set of solutions of ILPs (Eq. 4) for $S \in \mathcal{S}$, that is,

$$
Q^{x y}:=\left\{x \in \mathbb{Z}_{+}^{m}, y \in\{0,1\}^{n}: A x \geq B y+d, y=y(S) \text { for some } S \in \mathcal{S}\right\} .
$$

We now define, for each $(\bar{x}, \bar{y}) \in Q^{x y}$, the variable $z_{(\bar{x}, \bar{y})}$ of cost $c \bar{x}$. The resulting master LP is:

$$
\min \left\{\sum_{(\bar{x}, \bar{y}) \in Q^{x y}}(c \bar{x}) z_{(\bar{x}, \bar{y})}: \sum_{(\bar{x}, \bar{y}) \in Q^{x y}} \bar{y}_{i} z_{(\bar{x}, \bar{y})}=1, \quad i \in V, \quad z_{(\bar{x}, \bar{y})} \geq 0, \quad(\bar{x}, \bar{y}) \in Q^{x y}\right\} .
$$

This is easily seen to be equivalent to the LP (Eq. 11), since deleting for all $S \in \mathcal{S}$ the $z_{(\bar{x}, \bar{y})}$ variables with $\bar{y}=y(S)$ and $\cos t c \bar{x}>c(S)$ has no effect on the optimal solution. Thus:

Observation 3 For an IM game, the set of cost shares and the set of dual solutions to $L P$ (Eq. 12) coincide.

The pricing (column generation) problem for the LP (Eq. 12) amounts to optimizing over $Q^{x y}$. By the well-known equivalence between separation and optimization (see, e.g., [17]), this is strongly $\mathcal{N} \mathcal{P}$-hard for many IM games of interest since the OCSP is $\mathcal{N} \mathcal{P}$-hard, as we will see in the following. It is well-known (see, e.g., [4]) that pricing 
can sometimes be made more tractable by enlarging the set of variables in an appropriate way, without changing the set of constraints. This is clearly a relaxation, and, since it amounts to adding extra constraints in the primal problem (Eq. 3), it means that we may get a suboptimal cost share. The advantage, however, is that the pricing problem may become polynomially solvable, or at least solvable in pseudo-polynomial time. We give examples of this method in Sect. 6.

\subsection{Row generation}

The next method is based on the use of (strong) valid inequalities as cutting planes. The key is to interpret the Dantzig-Wolfe reformulation given in the previous subsection in terms of polyhedra and valid linear inequalities. To do this, it is helpful to define the following polyhedra.

The convex hull of integer solutions to the ILP (Eq. 10) is the polyhedron:

$$
P_{I}^{x}:=\operatorname{conv}\left\{x \in \mathbb{Z}_{+}^{m}: A x \geq B \mathbf{1}+d\right\} .
$$

The set of feasible solutions to the LP relaxation of Eq. 10 is the polyhedron:

$$
P^{x}:=\left\{x \in \mathbb{R}_{+}^{m}: A x \geq B \mathbf{1}+d\right\} .
$$

We also define an associated 'master' polyhedron in $(x, y)$-space:

$$
P_{I}^{x y}:=\operatorname{conv} Q^{x y}
$$

Note that $P_{I}^{x}$ can be obtained by intersecting $P_{I}^{x y}$ with the hyperplanes defined by $y_{i}=1$ for all $i \in V$, and then projecting the result onto $x$-space. That is:

$$
P_{I}^{x}=\operatorname{proj}_{x}\left(P_{I}^{x y} \cap\left\{(x, y) \in \mathbb{R}^{m+n}: y=\mathbf{1}\right\}\right) .
$$

For instance, in the case of the traveling salesman game, $P_{I}^{x}$ is the convex hull of the incidence vectors of the solutions of the traveling salesman problem on $G$, i.e., of the Hamiltonian circuits on $V \cup\{0\}, P^{x}$ is the set of the feasible solutions of the LP relaxation of the (customary) ILP formulation of traveling salesman given by Eq. 6 with $S=V$, and $P_{I}^{x y}$ is the convex hull of the vectors $(x, y)$, with $y$ incidence vector of a coalition $S \in \mathcal{S}$ and $x$ incidence vector of a Hamiltonian circuit on $S \cap\{0\}$.

Finally, we introduce the cone

$$
C^{x y}:=\text { cone } Q^{x y}
$$

and its projection onto $x$-space:

$$
C^{x}:=\operatorname{proj}_{x}\left(C^{x y} \cap\left\{(x, y) \in \mathbb{R}^{m+n}: y=\mathbf{1}\right\}\right) .
$$

Note that $P_{I}^{x y} \subseteq C^{x y}$ and therefore $P_{I}^{x} \subseteq C^{x} . C^{x y}$ and $C^{x}$ do not have the same intuitive interpretation as $P_{I}^{x y}$ and $P_{I}^{x}$, but they play a crucial role in our context, 
as shown below. In particular, $C^{x y}$ contains not only all vectors in $P_{I}^{x y}$, but also all vectors that can be written as $(\lambda x, \lambda y)$ for some $(x, y) \in P_{I}^{x y}$ and some $\lambda \in \mathbb{R}_{+}$. Then, $C^{x}$ is obtained by intersecting $C^{x y}$ with the $n$ hyperplanes $y_{i}=1$ for $i \in V$ and then projecting onto $x$-space.

We have the following lemma:

Lemma 1 The value of the optimal cost share is equal to $\min \left\{c x: x \in C^{x}\right\}$.

Proof Each variable $z_{S}$ in LP (Eq. 11) corresponds to a vector $(\bar{x}, \bar{y}) \in Q^{x y}$ with $\bar{y}=y(S)$ and $\bar{x}$ optimal solution of Eq. 4, i.e., $c \bar{x}=c(S)$. We observed above that the enlarged LP (Eq. 12) has the same value. Interpreting the $z_{(\bar{x}, \bar{y})}$ variables as coefficients in a conic combination of the points in $Q^{x y}$, there is a one-to-one correspondence between the set of solutions of LP (Eq. 12) and the set of points in the intersection of $C^{x y}$ with the hyperplanes $y_{i}=1$ for $i=1, \ldots, n$. Accordingly, since $C^{x}$ is the projection onto $x$-space of this intersection, the optimal value of Eq. 11 coincides with $\min \left\{c x: x \in C^{x}\right\}$.

Since $P_{I}^{x} \subseteq C^{x} \subseteq P^{x}$ when $d \geq 0$, for such games the value of the optimal cost share is no smaller than the lower bound obtained by solving the LP relaxation of Eq. 10 .

The above result can be stated in a rather different way using the idea of what we call assignable inequalities.

Definition 2 An inequality $\alpha x \geq \beta$ which is valid for $P_{I}^{x}$ is said to be assignable if there exists an inequality $\alpha x \geq \gamma y$ which is valid for $P_{I}^{x y}$, and such that $\sum_{i \in V} \gamma_{i}=\beta$.

In other words, an inequality valid for $P_{I}^{x}$ is assignable if it corresponds to a homogeneous inequality which is valid for $P_{I}^{x y}$.

Lemma $2 C^{x}$ is the set of points in $\mathbb{R}^{m}$ that satisfy all assignable inequalities.

Proof Clearly, a homogeneous inequality $\alpha x \geq \gamma y$ is valid for $P_{I}^{x y}$ if and only if it is valid for $C^{x y}$. Moreover, an inequality $\alpha x \geq \beta$ is valid for $C^{x}$ if and only if there exists an inequality $\alpha x \geq \gamma y$ which is valid for $C^{x y}$ such that $\sum_{i \in V} \gamma_{i}=\beta$.

The immediate implication of Lemmas 1 and 2 is:

Theorem 1 For an IM game, the value of the optimal cost share is equal to the minimum of cx subject to all assignable inequalities.

Corollary 1 For an IM game, the core is non-empty if and only if the optimal value of ILP (Eq. 4) for $S=V$ coincides with the lower bound obtained by minimizing cx subject to all assignable inequalities.

A proof of a result analogous to Theorem 1 was obtained independently in [13] for the class of games considered therein. Note that this theorem is not constructive, i.e., it does not provide a direct way to define a cost share given a set of assignable inequalities and the solution of the corresponding LP relaxation. This is achieved by the following method, which is the main practical contribution of the paper. 
Theorem 2 Consider an IM game, along with a set of assignable inequalities Dx $\geq f$ corresponding to a collection $D x \geq E y$ of homogeneous inequalities valid for $\bar{P}_{I}^{x y}$, with $f=E \mathbf{1}$. Suppose there is a separation algorithm for inequalities $D x \geq f$ that runs in time polynomial in $m, n$ and $\log \left|e_{\max }\right|$, where $e_{\max }$ is the largest entry in $(D, E)$ (in absolute value). Then a cost share with value

$$
\min \{c x: D x \geq f\}
$$

can be found in time polynomial in $m, n$ and $\log \left|e_{\max }\right|$.

Proof If we can separate in polynomial time over $D x \geq f$, then by the equivalence between optimization and separation (see, e.g., [17]) we can solve LP (Eq. 13) in polynomial time, finding a basic solution $\pi^{*}$ of the dual of Eq. 13, given by:

$$
\max \left\{f \pi: D^{T} \pi=c, \pi \geq 0\right\} .
$$

Since $\pi^{*}$ is basic, at most $p \leq m$ of its components are strictly positive. Assuming for notational convenience that the first $p$ components of $\pi^{*}$ are strictly positive, we define the following solution of Eq. 3:

$$
w_{i}^{*}:=\sum_{h=1}^{p} e_{h i} \pi_{h}^{*}, \quad i \in V
$$

The value of $w^{*}$ is

$$
\sum_{i \in V} w_{i}^{*}=\sum_{i \in V} \sum_{h=1}^{p} e_{h i} \pi_{h}^{*}=\sum_{h=1}^{p}\left(\sum_{i \in V} e_{h i}\right) \pi_{h}^{*}=f \pi^{*},
$$

equal to the optimal value of Eqs. 13 and 14. In order to show that $w^{*}$ is a cost share, note that, for any $S \in \mathcal{S}, \pi^{*}$ is a feasible solution of

$$
\max \left\{(E y(S)) \pi: D^{T} \pi=c, \pi \geq 0\right\},
$$

which is the dual of

$$
\min \{c x: D x \geq E y(S)\}
$$

whose value is not larger than $c(S)$ since all inequalities are valid for ILP (Eq. 4). This implies

$$
\sum_{i \in S} w_{i}^{*}=\sum_{i \in S} \sum_{h=1}^{p} e_{h i} \pi_{h}^{*}=\sum_{h=1}^{p}\left(\sum_{i \in S} e_{h i}\right) \pi_{h}^{*} \leq c(S),
$$

i.e., $w^{*}$ is a cost share. 
The proof of the above result is based on the ellipsoid method, but in practice one can use a standard simplex-based cutting plane method.

We conclude the section by showing the following result:

Theorem 3 If $d \geq 0$, then $C^{x} \subseteq P^{x}$, and therefore the value of the optimal cost share is at least as large as the value of the LP relaxation of $E q .4$ for $S=V$.

Proof We show that every inequality valid for $P^{x}$ is valid also for $C^{x}$. If an inequality $\alpha x \geq \beta$ is valid for $P^{x}$, then there exists $\lambda \in \mathbb{R}_{+}^{p}$ such that $\alpha \geq \lambda A$ and $\beta=\lambda(B \mathbf{1}+d)$. This implies that the inequality $\alpha x \geq \gamma y+\rho$ with $\gamma=\lambda B$ and $\rho=\lambda d$ is implied by the original system $A x \geq B y+d$ (and by non-negativity) and therefore valid for $P_{I}^{x y}$. Since $\rho \geq 0$, we can weaken the inequality $\alpha x \geq \gamma y+\rho$ to $\alpha x \geq \gamma y+\rho y_{i}$ for some arbitrary $i \in V$. Recalling the arguments in the proof of Lemma 2, this weaker inequality is valid for $P_{I}^{x y}$ and therefore, being homogeneous, is also valid for $C^{x y}$. Then, since $\rho+\sum_{i \in V} \gamma_{i}=\beta$, inequality $\alpha x \geq \beta$ is valid for $C^{x}$.

\subsection{Combined column and row generation}

In some cases, it may pay to combine both of the above ideas. Let $D x \geq f$ be, as before, a family of assignable inequalities for which efficient separation algorithms are known. Consider the following LP, obtained by linking together the variables and constraints of this linear system with those of the column generation approach:

$$
\begin{aligned}
& \min \left\{\sum_{(\bar{x}, \bar{y}) \in Q^{x y}}(c \bar{x}) z_{(\bar{x}, \bar{y})}: \quad \sum_{(\bar{x}, \bar{y}) \in Q^{x y}} \bar{y}_{i} z_{(\bar{x}, \bar{y})}=1, \quad i \in V,\right.
\end{aligned}
$$

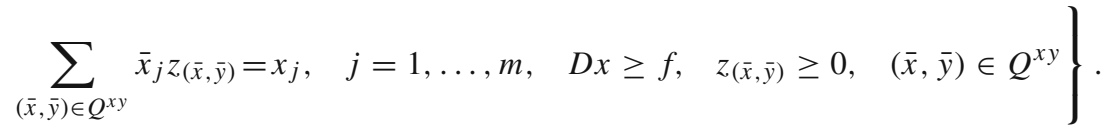

An LP of this type is called an 'explicit master' in [6]. It can be solved by a combination of row and column generation, i.e., cut-and-price. The resulting lower bound is at least as strong as the bound obtained by either cutting or pricing alone. The following theorem demonstrates that such a cut-and-price algorithm can again be used to derive cost shares.

Theorem 4 Consider an IM game and a set of assignable inequalities Dx $\geq f$ as in the statement of Theorem 2. Given a dual solution of LP (Eq. 15), a cost share with at least the same value can be found in time polynomial in $m, n$ and $\log \left|e_{\max }\right|$.

Proof The dual of Eq. 15 reads:

$$
\max \left\{\sum_{i \in V} w_{i}+f \pi: \sum_{i \in V} \bar{y}_{i} w_{i}+\sum_{j=1}^{m} \bar{x}_{j} \rho_{j} \leq c \bar{x}, \quad(\bar{x}, \bar{y}) \in Q^{x y}, \quad D^{T} \pi=\rho, \quad \pi \geq 0\right\} .
$$

Given a feasible solution $\left(w^{*}, \rho^{*}, \pi^{*}\right)$ of Eq. 16, we first define a solution $\left(w^{*}, \rho^{*}, \pi^{* *}\right)$ which is at least as good and in which at most $p \leq m$ of $\pi^{* *}$ are 
strictly positive by finding a basic solution of the following LP:

$$
\max \left\{f \pi: D^{T} \pi=\rho^{*}, \pi \geq 0\right\} .
$$

This can be obtained in time polynomial in $m$ (see again, e.g., [17]) without reading the original $\pi^{*}$ vector, whose number of nonzero components may be exponential in $m$.

Assuming again that the first $p$ components of $\pi^{* *}$ are strictly positive, we define the following solution of Eq. 3 of the same value as $\left(w^{*}, \rho^{*}, \pi^{* *}\right)$ :

$$
w_{i}^{* *}:=w_{i}^{*}+\sum_{h=1}^{p} e_{h i} \pi_{h}^{* *}, \quad i \in V .
$$

In order to verify that $w^{* *}$ is feasible for Eq. 3, it is enough to observe that, for each $S \in \mathcal{S}$, letting $\bar{x}$ be an optimal solution of ILP (Eq. 4) for $S$, with $c \bar{x}=c(S)$ and $D \bar{x} \geq E y(S)$,

$$
\begin{aligned}
\sum_{i \in S} w_{i}^{* *} & =\sum_{i \in S} w_{i}^{*}+\sum_{i \in S} \sum_{h=1}^{p} e_{h i} \pi_{h}^{* *} \\
& \leq \sum_{i \in S} w_{i}^{*}+\sum_{h=1}^{p}\left(\sum_{i \in S} e_{h i}\right) \pi_{h}^{* *} \leq \sum_{i \in S} w_{i}^{*}+\sum_{h=1}^{p}\left(\sum_{j=1}^{m} d_{h j} \bar{x}_{j}\right) \pi_{h}^{* *} \\
& =\sum_{i \in S} w_{i}^{*}+\sum_{j=1}^{m}\left(\sum_{h=1}^{p} d_{h j} \pi_{h}^{* *}\right) \bar{x}_{j} \\
& =\sum_{i \in S} w_{i}^{*}+\sum_{j=1}^{m} \rho_{j}^{*} \bar{x}_{j} \leq c \bar{x},
\end{aligned}
$$

where the last equation and inequality follow from the constraints in Eq. 16.

\subsection{Handling non-negative weights}

So far, we have concentrated on the case in which the weights $w_{i}$ are allowed to be negative. The methods that we have introduced above can be easily adjusted to handle the case in which weights are required to be non-negative.

If weights must be non-negative, this is the LP whose solution yields an optimal cost share:

$$
\max \left\{\sum_{i \in V} w_{i}: \sum_{i \in S} w_{i} \leq c(S), \quad S \in \mathcal{S}, \quad w_{i} \geq 0, \quad i \in V\right\} .
$$

The difference is that now in the dual we have covering rather than partitioning constraints:

$$
\min \left\{\sum_{S \in \mathcal{S}} c(S) z_{S}: \sum_{S \ni i} z_{S} \geq 1, \quad i \in V, z_{S} \geq 0, \quad S \in \mathcal{S}\right\} .
$$


We call an IM game for which weights must be non-negative a non-negative IM game. All the results in Sect. 3 can easily be adapted to this case. In particular, a characterization of the value of an optimal cost share is given in the following. Let $C^{x y}$ be defined as before and

$$
\tilde{C}^{x}:=\operatorname{proj}_{x}\left(C^{x y} \cap\left\{(x, y) \in \mathbb{R}^{m+n}: y \geq \mathbf{1}\right\}\right) .
$$

An assignable inequality $\alpha x \geq \beta$ associated with a valid inequality $\alpha x \geq \gamma y$ for $P_{I}^{x y}$ is said to be non-negative if $\gamma \geq 0$.

Lemma 3 For a non-negative IM game, the value of the optimal cost share is equal to $\min \left\{c x: x \in \tilde{C}^{x}\right\}$.

Lemma $4 \tilde{C}^{x}$ is the set of points in $\mathbb{R}^{m}$ that satisfy all non-negative assignable inequalities.

Proof Letting $G, H$ be such that $C^{x y}=\left\{(x, y) \in \mathbb{R}^{m+n}: G x \geq H y\right\}$, an inequality $\alpha x \geq \beta$ is valid for $\tilde{C}^{x}$ if and only if it can be obtained as a conic combination of the inequalities $G x-H y \geq 0, y \geq \mathbf{1}, 0 \geq-1$, i.e., there exist non-negative multiplier vectors $\lambda, \gamma \geq 0$ such that $\lambda G=\alpha, \lambda H=\gamma$, and $\beta \leq \gamma \mathbf{1}$. Accordingly, $\alpha x \geq \beta$ is valid for $\tilde{C}^{\bar{x}}$ if and only if it corresponds to a valid inequality for $P_{I}^{x y}$ of the form $\alpha x \geq \gamma y$ with $\gamma \geq 0$.

Theorem 5 For a non-negative IM game, the value of the optimal cost share is equal to the minimum of $c x$ subject to all non-negative assignable inequalities.

Theorem 6 Consider a non-negative IM game, along with a set of non-negative assignable inequalities $D x \geq f$ as in the statement of Theorem 2. A cost share with value

$$
\min \{c x: D x \geq f\}
$$

can be found in time polynomial in $m, n$ and $\log \left|e_{\max }\right|$.

Proof Identical to the proof of Theorem 2, noting that non-negativity of $E$ and $\pi^{*}$ implies non-negativity of $w^{*}$.

Although in general the optimal value of Eq. 17 is smaller than that of LP (Eq. 3), there is a wide class of games for which the two values coincide.

Observation 4 For a monotonic game with $\mathcal{S}=2^{V} \backslash\{\emptyset\}$, given a feasible solution $w^{*}$ of Eq. 3, the solution $\bar{w}_{i}:=\max \left\{w_{i}^{*}, 0\right\}$ for $i \in V$ is also feasible.

Proof Consider a feasible solution $w^{*}$ of Eq. 3 with $w_{i}^{*}<0$ for some $i \in V$. The proof follows by showing that setting $w_{i}^{*}:=0$ yields another feasible solution. If there exists no $S \in \mathcal{S}$ such that $i \in S$ and $\sum_{j \in S} w_{j}^{*}=c(S)$, we can set $w_{i}^{*}:=\min _{S \in \mathcal{S}: i \in S} c(S)-$ $\sum_{j \in S \backslash\{i\}} w_{j}^{*}$ maintaining feasibility (and increasing the solution value). If this yields $w_{i}^{*} \geq 0$ we are done. Otherwise, consider $S \in S$ such that $i \in S$ and $\sum_{j \in S} w_{j}^{*}=c(S)$. Since $w_{i}^{*}<0$ and $c(S \backslash\{i\}) \leq c(S)$, we have $\sum_{j \in S \backslash\{i\}} w_{j}^{*}>c(S \backslash\{i\})$, a contradiction to the initial assumption on the feasibility of $w^{*}$. 
Corollary 2 For a monotonic game with $\mathcal{S}=2^{V} \backslash\{\emptyset\}$, the values of Eqs. 3 and 17 coincide, and every optimal cost share $w^{*}$ satisfies $w_{i}^{*} \geq 0$ for $i \in V$.

In particular, given a feasible solution of Eq. 3, Observation 4 shows how to construct immediately a feasible solution of Eq. 17 whose value is not smaller.

Corollary 2 applies to the covering and facility location games, as well as to the metric traveling salesman and vehicle routing games. As for the metric two-matching and unrooted traveling salesman games, it is possible to show that imposing non-negativity makes no difference, but this requires an ad-hoc proof that is not given here.

\section{Application to covering and facility location games}

We show how the results concerning the OCSP in [7] for covering games, and in [14] for facility location games, arise as fairly simple corollaries of the above results on $P^{x}, P_{I}^{x}$ and $C^{x}$.

Indeed, [7] proves that, for an IM game in which $\mathcal{S}=2^{V} \backslash\{\emptyset\}, B$ is the identity matrix, $A$ is binary and $d=0$, the value of the optimal cost share coincides with that of the LP relaxation of Eq. 4 for $S=V$. This can be seen as a special case of the above results, combined with the following observation, whose proof (using ideas analogous to those in [7]) also suggests why the result does not hold if any of the above conditions is relaxed.

Observation 5 For an IM game in which $\mathcal{S}=2^{V} \backslash\{\emptyset\}, B$ is the identity matrix, $A$ is binary and $d=0, C^{x y}=\left\{(x, y) \in \mathbb{R}_{+}^{m+n}: A x \geq y\right\}$.

Proof Let $D:=\left\{(x, y) \in \mathbb{R}^{m+n}: A x \geq y\right\}$. Clearly, $C^{x y} \subseteq D$. In order to show $D \subseteq C^{x y}$, we consider $(x, y)^{\prime} \in D$ and express it as a conic combination of the points in $Q^{x y}$. Consider $j$ such that $x_{j}^{\prime}>0$ and let $S:=\left\{i \in V: a_{i j}=1\right.$ and $\left.y_{i}^{\prime}>0\right\}$. Take the vector $(x, y)^{\prime} \in Q^{x y}$, whose components at 1 are $x_{j}^{\prime}$ and $y_{i}^{\prime}$ for $i \in S$, let $\lambda:=\min \left\{x_{j}^{\prime}, \min _{i \in S} y_{i}^{\prime}\right\}$ and define $(x, y)^{\prime \prime}:=(x, y)^{\prime}-\lambda(x, y)^{\prime}$. We claim $(x, y)^{\prime \prime} \in D$. Indeed, if this was false we would have $\sum_{k=1}^{m} a_{i k} x_{k}^{\prime \prime}<y_{i}^{\prime \prime}$, which is easily seen to imply $\sum_{k=1}^{m} a_{i k} x_{k}^{\prime}<y_{i}^{\prime}$, a contradiction to $(x, y)^{\prime} \in D$. Therefore, $(x, y)^{\prime \prime}$ is in $D$ and has at least one additional zero component with respect to $(x, y)^{\prime}$. Iterating the procedure until the null vector is obtained yields the desired conic combination.

For the facility location games mentioned in Sect. 2.2, the authors of [14] showed that the value of the optimal cost share is given by the LP relaxation of Eq. 5 for $S=V$ in which constraints $\left(v_{j}, u_{1 j}, \ldots, u_{n j}\right) \in F_{j}$ are replaced by $\left(v_{j}, u_{1 j}, \ldots, u_{n j}\right) \in$ cone $F_{j}$ for $j=1, \ldots, q$. Also this result can be seen as a special case of ours by combining the results of Sect. 3.2 with the following observation:

Observation 6 For an IM game with $\mathcal{S}=2^{V} \backslash\{\emptyset\}$ and $d=0$ defined by Eq. 5, $C^{x y}=$ $\left\{(v, u, y) \in \mathbb{R}^{q+n q+n}: \sum_{j=1}^{q} u_{i j}=y_{i}(i \in V),\left(v_{j}, u_{1 j}, \ldots, u_{n j}\right) \in\right.$ cone $F_{j}(j=$ $1, \ldots, q)\}$. 
Proof Let $D:=\left\{(v, u, y) \in \mathbb{R}^{q+n q+n}: \sum_{j=1}^{q} u_{i j}=y_{i}(i \in V),\left(v_{j}, u_{1 j}, \ldots, u_{n j}\right) \in\right.$ cone $\left.F_{j}(j=1, \ldots, q)\right\}$ and note that, in this case,

$$
Q^{x y}=\left\{(v, u) \in \mathbb{Z}_{+}^{q+n q}, y \in\{0,1\}^{n}: \sum_{j=1}^{q} u_{i j}=y_{i}, \quad i \in V,\right.
$$

In order to see that cone $Q^{x y}=D$, note first that cone $Q^{x y} \subseteq D$ is obvious. Conversely, given a point $(v, u, y)^{\prime} \in D$ with $\left(v_{j}^{\prime}, u_{1 j}^{\prime}, \ldots, u_{n j}^{\prime}\right)=\sum_{R \in \mathcal{R}_{j}} \lambda_{R}^{\prime}(1, y(R))$ for $j=1, \ldots, q$, we have $(v, u, y)^{\prime}=\sum_{j=1}^{q} \sum_{R \in \mathcal{R}_{j}} \lambda_{R}(v, u, y)^{R}$, where, for $j=$ $1, \ldots, q$ and $R \in \mathcal{R}_{j}$, the vector $(v, u, y)^{R} \in Q^{x y}$ has components at 1 given by $v_{j}$ and $u_{i j}, y_{i}$ for $i \in R$.

\section{Application to the unrooted traveling salesman game}

In this section, we apply the results of Sect. 3 to the two-matching and unrooted traveling salesman games. The results are fairly straightforward, but they serve to illustrate the techniques and provide a good basis for the analysis of the more complex games in Sect. 6.

A simple and well-known property that turns out to be useful in the study of these games is:

Observation 7 A constraint in Eq. 3 for a given set $S$ is redundant if there exists a partition of $S$ into two sets $S_{1}, S_{2} \in \mathcal{S}$ such that $c\left(S_{1}\right)+c\left(S_{2}\right) \leq c(S)$.

Proof Summing together the constraints (Eq. 3) for $S_{1}$ and $S_{2}$ yields:

$$
\sum_{i \in S} w_{i} \leq c\left(S_{1}\right)+c\left(S_{2}\right)
$$

that dominates the inequality in Eq. 3 associated with $S$.

This yields the following corollary:

Corollary 3 A cost share is feasible for the unrooted traveling salesman game if and only if it is feasible for the two-matching game (and the two cost shares have the same value).

Proof In the case of the two-matching game, $c(S)$ is the minimum cost of a two-matching on the vertices in $S$. If this two-matching consists of two or more disjoint circuits, then the associated constraint is redundant by Observation 7. Since the minimum cost two-matching on $S$ costs no more than the minimum cost Hamiltonian circuit on $S$, the LP (Eq. 3) is unchanged if, for every set $S$, we replace $c(S)$ with the cost of the minimum cost Hamiltonian circuit on $S$. 
As already observed in [10], the OCSP for the unrooted traveling salesman game (and therefore, by Corollary 3 , for the two-matching game) can be solved in polynomial time. Moreover, it is easy to see that for the two-matching game testing non-emptyness of the core as well as if a given vector is in the core can be done in polynomial time. On the other hand, the following holds for the unrooted traveling salesman game:

Observation 8 Testing non-emptyness of the core for the unrooted traveling salesman game is strongly $\mathcal{N} \mathcal{P}$-complete, even in the metric case.

Proof Since the OCSP can be solved in polynomial time, the problem is in $\mathcal{N} \mathcal{P}$. We show $\mathcal{N} \mathcal{P}$-completeness by a reduction from the problem of testing if a graph $H=(V, F)$ with $V=\{1, \ldots, n\}$ is Hamiltonian. Consider the unrooted traveling salesman game associated with the complete graph $G$ with vertex set $V$ and where the cost of each edge $(i, j)$ is given by 1 if $(i, j) \in F$ and 2 if $(i, j) \notin F$, noting that these costs satisfy the triangle inequality. We have that $H$ is Hamiltonian if and only if $c(V)=n$. This can be tested as follows. First of all, we compute the optimal two-matching value for $G$. If it is larger than $n$, then clearly $c(V)>n$. Otherwise, the optimal two-matching value is $n$, and, by Corollary $3, w_{i}=1$ for $i \in V$ is an optimal cost share of value $n$ for both the two-matching and the unrooted traveling salesman game. Then, $c(V)=n$ if and only if the core of the unrooted traveling salesman game is non-empty. Therefore, by testing non-emptyness of the core in polynomial time we could test if $H$ is Hamiltonian in polynomial time.

For the two-matching game, we have

$$
P^{x}=\left\{x \in[0,1]^{|E|}: \sum_{e \in \delta(i)} x_{e}=2, \quad i \in V\right\}
$$

and

$$
P_{I}^{x}=\operatorname{conv}\left\{x \in\{0,1\}^{|E|}: \sum_{e \in \delta(i)} x_{e}=2, \quad i \in V\right\} .
$$

It is well known that $P^{x} \neq P_{I}^{x}$ in this case. A complete description of $P_{I}^{x}$ was given in [8]. Together with the bound constraints and the degree equations, the following blossom inequalities describe the convex hull $P_{I}^{x}$ :

$$
\sum_{e \in \delta(R) \backslash F} x_{e} \geq \sum_{e \in F} x_{e}-|F|+1, \quad R \subset V, F \subset \delta(R),|F| \text { odd } .
$$

Moreover, a necessary and sufficient condition for a blossom inequality to induce a facet of $P_{I}^{x}$ is that the edges in $F$ are vertex disjoint [19].

It turns out that $P_{I}^{x} \subset C^{x} \subset P^{x}$, i.e., only a subset of the blossom inequalities are assignable:

Theorem 7 For the two-matching and unrooted traveling salesman games, a facetinducing blossom inequality (Eq. 20) is assignable if and only if $|F|=1$. 
Proof Restricting to the case $|F|=1$, the inequalities read

$$
\sum_{e \in \delta(R) \backslash\{f\}} x_{e}-x_{f} \geq 0, \quad R \subset V, f \in \delta(R),
$$

and are easily checked to be valid for $P_{I}^{x y}$. Now, suppose $q=|F|>1$ and odd. Without loss of generality suppose that $R$ contains vertices $1, \ldots, q$ but not $q+1, \ldots, 2 q$, and that the edges in $F$ are $\{(i, q+i): i=1, \ldots, q\}$. The inequality (Eq. 20) is assignable if and only if there exists a vector $\gamma \in \mathbb{R}^{n}$ such that $\sum_{e \in \delta(R) \backslash F} x_{e}-\sum_{e \in F} x_{e} \geq$ $\sum_{i \in V} \gamma_{i} y_{i}$ is valid for $P_{I}^{x y}$ and $\sum_{i \in V} \gamma_{i}=1-q$. We show that this cannot be the case. For any $j=1, \ldots, q-2$, consider the circuit visiting vertices $j, j+1, q+j+1$ and $q+j$ in that order. Since each of these circuits satisfies $\sum_{e \in \delta(R) \backslash F} x_{e}-\sum_{e \in F} x_{e}=-2$, we must have:

$$
\gamma_{j}+\gamma_{j+1}+\gamma_{q+j}+\gamma_{q+j+1} \leq-2, \quad j=1, \ldots, q-2 .
$$

Similarly, a consideration of any circuit which passes through vertices $q-1, q$ and $2 q-1, \ldots, n$, but which only crosses $\delta(R)$ in edges $(q-1,2 q-1)$ and $(q, 2 q)$, shows that:

$$
\gamma_{q-1}+\gamma_{q}+\sum_{j=2 q-1}^{n} \gamma_{j} \leq-2
$$

Finally, a consideration of any circuit which passes through vertices $1, q, q+1$, and $2 q, \ldots, n$, but which only crosses $\delta(R)$ in edges $(1, q+1)$ and $(q, 2 q)$, shows that:

$$
\gamma_{1}+\gamma_{q}+\gamma_{q+1}+\sum_{j=2 q}^{n} \gamma_{j} \leq-2
$$

Summing together the $q-2$ inequalities (Eq. 22) and the inequalities (Eqs. 23, 24), and dividing by two gives $\sum_{i \in V} \gamma_{i} \leq-q$, which is a contradiction to $\sum_{i \in V} \gamma_{i}=1-q$.

This gives the following corollary:

Corollary 4 For the two-matching and unrooted traveling salesman games, the value of the optimal cost share is equal to the value of the LP relaxation of ILP (Eq. 9) for $S=V$ augmented by constraints (Eq. 21).

This corollary can also be proved in a rather different way by using a result of [34], which states that $x$ is a conic combination of circuits in $\mathcal{C}$ if and only if $x$ is non-negative and satisfies (Eq. 21).

Since the separation problem for the inequalities (Eq. 21) can be easily solved in polynomial time via maximum flow methods, the LP mentioned in Corollary 4 can be solved in polynomial time. This gives an alternative way to efficiently compute an optimal cost share for the two games considered, though still based on the ellipsoid 
method. On the other hand, we do not know if there is a combinatorial algorithm that does the job.

In conclusion, then, the optimal cost share for the two-matching game (and therefore the unrooted traveling salesman game) has a value which lies between the cost of an optimal two-matching and the cost of an optimal fractional two-matching. It is easy to show that these three values can all be different even in the metric case.

\section{Application to traveling salesman and vehicle routing games}

In this section, we examine the traveling salesman and vehicle routing games. These are considerably more complicated than the preceding games. Recall that in this case $0 \notin V$ is the depot. We have the following counterpart of Corollary 3, implied by Lemma 7 as well:

Corollary 5 If $Q \geq \sum_{i \in V} q_{i}$, a cost share is feasible for the traveling salesman game if and only if it is feasible for the vehicle routing game (and the two cost shares have the same value).

Proof For the vehicle routing game, the constraint for a coalition $S$ is non-redundant if and only if $c(S)$ does not correspond to the union of two or more circuits, but to a single circuit passing through the depot. Since the minimum cost vehicle routing solution on $S \cup\{0\}$ costs no more than the minimum cost Hamiltonian circuit on $S \cup\{0\}$, the LP (Eq. 3) is unchanged if, for every set $S$, we replace $c(S)$ with the cost of the minimum cost Hamiltonian circuit on $S$.

For these games, [13] proved that the OCSP is strongly $\mathcal{N} \mathcal{P}$-hard, even in the metric case. Moreover, [29] proved that testing non-emptyness of the core is also strongly $\mathcal{N P}$-hard (noting that, to the best of our knowledge, the problem is not known to be in $\mathcal{N P}$ ), and [2] extended this result to the metric case. In [9] it is shown that, for the traveling salesman game, the OCSP value is at least equal to the well-known HeldKarp lower bound in the metric case (the paper only mentions Euclidean distances, but the proof only requires distances to satisfy the triangle inequality).

\subsection{The traveling salesman game}

Since computing the optimal cost share is strongly $\mathcal{N} \mathcal{P}$-hard for the traveling salesman game, we propose the use of the column or row generation methods to compute good cost shares.

In the column generation approach, there is one column for every circuit which passes through the depot vertex. We could enlarge the set of columns to allow one column for every circuit, whether or not it included the depot. We would then have the same LP as for the two-matching and unrooted traveling salesman games, and therefore the pricing subproblem would become polynomially solvable. Another possibility would be to have one column for every closed walk containing the depot, as in the so-called $n$-path relaxation of the TSP [22]. The resulting pricing problem could then be solved by dynamic programming. 
However, we believe that the row generation approach is more promising, because we can exploit the considerable research which has been done into polyhedra associated with the TSP. Indeed, we have:

$P^{x}=\left\{x \in[0,1]^{|E|}: \sum_{e \in \delta(i)} x_{e}=2, \quad i \in V \cup\{0\}, \sum_{e \in \delta(R)} x_{e} \geq 2, \quad R \subseteq V, R \neq \emptyset\right\}$.

$P^{x}$ is the well-known subtour elimination polytope, whereas $P_{I}^{x}$ is the symmetric traveling salesman polytope (see, e.g., [26]).

From formulation (Eq. 6), it is obvious that:

Observation 9 For the traveling salesman game, the degree equations $\sum_{e \in \delta(i)} x_{e}=2$ are assignable for every $i \in V$ (equivalently, the inequalities $\sum_{e \in \delta(i)} x_{e} \geq 2$ and $\sum_{e \in \delta(i)} x_{e} \leq 2$ are assignable).

Observation 10 For the traveling salesman game, the subtour elimination constraints $\sum_{e \in \delta(R)} x_{e} \geq 2$ are assignable for every $R \subseteq V, R \neq \emptyset$.

It is possible to show that the degree equation for vertex 0 is not assignable (specifically, the inequality $\sum_{e \in \delta(0)} x_{e} \leq 2$ is not assignable). Since this degree equation is valid for $P^{x}$ but not for $C^{x}$, for the traveling salesman game we do not have $C^{x} \subseteq P^{x}$. This is to be expected, recalling Theorem 3 , since $d \nsucceq 0$.

We now consider another well-known class of facet-inducing inequalities for the TSP, the so-called clique tree inequalities of [20]. A clique-tree consists of a family of vertex sets $H_{1}, \ldots, H_{r}$, called handles, and another family of vertex sets $T_{1}, \ldots, T_{s}$, called teeth, such that:

- no two handles intersect,

- no two teeth intersect,

- the intersection graph (i.e., the graph with a vertex for each handle, a vertex for each tooth, and an edge between two vertices if and only if the corresponding handle and tooth intersect) is a tree,

- for each handle $H_{i}$, the number of teeth $t_{i}$ intersecting it is odd and at least three,

- each tooth contains at least one vertex which also lies in a handle, and at least one vertex which does not lie in any handle.

The associated clique tree inequality can be written as:

$$
\sum_{i=1}^{r} \sum_{e \in \delta\left(H_{i}\right)} x_{e}+\sum_{j=1}^{s} \sum_{e \in \delta\left(T_{j}\right)} x_{e} \geq 2 \sum_{i=1}^{r} t_{i}+s+1
$$

In the special case where we have only one handle, the clique tree inequalities reduce to the comb inequalities [18]:

$$
\sum_{e \in \delta(H)} x_{e}+\sum_{j=1}^{s} \sum_{e \in \delta\left(T_{j}\right)} x_{e} \geq 3 s+1
$$


Moreover, the comb inequalities for which $\left|T_{j}\right|=2$ for all $j$ are equivalent to the blossom inequalities discussed in the previous section.

In general, the clique tree inequalities are not assignable. However, if we weaken them by adding a suitable multiple of the degree inequality $\sum_{e \in \delta(0)} x_{e} \geq 2$, they become assignable. This is shown in the following theorem.

Theorem 8 For the traveling salesman game, the following weakened clique tree inequalities are assignable:

$$
\sum_{i=1}^{r} \sum_{e \in \delta\left(H_{i}\right)} x_{e}+\sum_{j=1}^{s} \sum_{e \in \delta\left(T_{j}\right)} x_{e}+\left\lfloor\frac{s}{2}\right\rfloor \sum_{e \in \delta(0)} x_{e} \geq 2 \sum_{i=1}^{r} t_{i}+2 s
$$

if the root does not lie in a tooth, and

$$
\sum_{i=1}^{r} \sum_{e \in \delta\left(H_{i}\right)} x_{e}+\sum_{j=1}^{s} \sum_{e \in \delta\left(T_{j}\right)} x_{e}+\left(\left\lfloor\frac{s}{2}\right\rfloor-1\right) \sum_{e \in \delta(0)} x_{e} \geq 2 \sum_{i=1}^{r} t_{i}+2 s-2
$$

if the root lies in a tooth.

Proof Let $W$ be an arbitrary set of vertices containing exactly one vertex from each non-empty intersection $H_{i} \cap T_{j}$ and, for each tooth, exactly one vertex which does not lie in any handle. Note that there are $\sum_{i=1}^{r} t_{i}$ vertices of the first type and $s$ of the second type, so $|W|=\sum_{i=1}^{r} t_{i}+s$. In [1] it is shown that the following inequality is satisfied by the incidence vector of any circuit (not necessarily one passing through the root):

$$
\sum_{i=1}^{r} \sum_{e \in \delta\left(H_{i}\right)} x_{e}+\sum_{j=1}^{s} \sum_{e \in \delta\left(T_{j}\right)} x_{e} \geq \sum_{i \in W} \sum_{e \in \delta(i)} x_{e}-s+1 .
$$

(Actually, [1] presented these inequalities in a different form, but her inequalities can easily be shown to be equivalent to ours, using the identity $\sum_{v \in S} \sum_{e \in \delta(v)} x_{e}=$ $\left.2 \sum_{e \in E(S)} x_{e}+\sum_{e \in \delta(S)} x_{e}.\right)$

The inequalities (Eq. 29) are clearly valid for $P_{I}^{x y}$, but are not homogeneous. To make them homogeneous, we add a suitable multiple of the inequality $\sum_{e \in \delta(0)} x_{e} \geq 2$ (which is clearly valid for $P_{I}^{x y}$ ). This gives:

$$
\sum_{i=1}^{r} \sum_{e \in \delta\left(H_{i}\right)} x_{e}+\sum_{j=1}^{s} \sum_{e \in \delta\left(T_{j}\right)} x_{e}+\left\lfloor\frac{s}{2}\right\rfloor \sum_{e \in \delta(0)} x_{e} \geq \sum_{i \in W} \sum_{e \in \delta(i)} x_{e} .
$$

Since $\sum_{e \in \delta(i)} x_{e}=2 y_{i}$ holds for all $i \in V$ and for all points in $P_{I}^{x y}$, we have:

$$
\sum_{i=1}^{r} \sum_{e \in \delta\left(H_{i}\right)} x_{e}+\sum_{j=1}^{s} \sum_{e \in \delta\left(T_{j}\right)} x_{e}+\left\lfloor\frac{s}{2}\right\rfloor \sum_{e \in \delta(0)} x_{e} \geq 2 \sum_{i \in W} y_{i}
$$


when the root does not lie in $W$ and

$$
\sum_{i=1}^{r} \sum_{e \in \delta\left(H_{i}\right)} x_{e}+\sum_{j=1}^{s} \sum_{e \in \delta\left(T_{j}\right)} x_{e}+\left(\left\lfloor\frac{s}{2}\right\rfloor-1\right) \sum_{e \in \delta(0)} x_{e} \geq 2 \sum_{i \in W \backslash\{0\}} y_{i}
$$

when the root lies in $W$. The result follows from the fact that $|W|=\sum_{i=1}^{r} t_{i}+s$.

In the special case of comb inequalities we get:

Corollary 6 For the traveling salesman game, the weakened comb inequalities

$$
\sum_{e \in \delta(H)} x_{e}+\sum_{j=1}^{s} \sum_{e \in \delta\left(T_{j}\right)} x_{e}+\left\lfloor\frac{s}{2}\right\rfloor \sum_{e \in \delta(0)} x_{e} \geq 4 s
$$

and

$$
\sum_{e \in \delta(H)} x_{e}+\sum_{j=1}^{s} \sum_{e \in \delta\left(T_{j}\right)} x_{e}+\left(\left\lfloor\frac{s}{2}\right\rfloor-1\right) \sum_{e \in \delta(0)} x_{e} \geq 4 s-2
$$

are assignable if the root does not lie and lies in a tooth, respectively.

Note that the comb inequality with three teeth does not have to be weakened if the root lies in a tooth. Thus, these special comb inequalities are facet-inducing as well as assignable, like the subtour elimination inequalities.

Corollary 7 For the traveling salesman game, a cost share exists whose value is at least the value of the LP relaxation of the traveling salesman problem with non-negativity constraints, degree equations for $i \in V$, subtour elimination constraints, and weakened clique tree inequalities.

However, the complexity of separation of the weakened clique tree inequalities is unknown. Therefore, Corollary 7 does not yield a constructive algorithm to find the desired cost share. However, heuristics for separation are not difficult to construct. Moreover, in the special case where there is only one handle and $\left|T_{j}\right|=2$ for all $j$, the weakened comb inequalities become similar to blossom inequalities, and it is not difficult to show that they can be separated in polynomial time [30]. Thus, we obtain the following result:

Corollary 8 For the traveling salesman game, we can find in polynomial time a cost share whose value is at least equal to the value of the LP relaxation of the traveling salesman problem with non-negativity constraints, degree equations for $i \in V$, subtour elimination constraints, and weakened blossom inequalities (i.e., inequalities (Eq. 31) with $\left|T_{j}\right|=2$ for all $j$ and $0 \in \bigcup_{j} T_{j}$ and inequalities (Eq. 30) with $\left|T_{j}\right|=2$ for all $j$ and $0 \notin \bigcup_{j} T_{j}$ ).

We remark that some other facet-inducing and assignable inequalities were presented in [13]: they belong to the class of so-called 2-brush inequalities of [27]. 


\subsection{The vehicle routing game}

The column generation approach is most naturally applied to the vehicle routing game. Note that, for this game, Observation 7 implies that we can disregard all coalitions $S$ apart from those with $\sum_{i \in S} q_{i} \leq Q$. That is, we only need one column for each feasible route for a single vehicle. The resulting set partitioning LP is well known in the vehicle routing literature (see, e.g., [4]). As mentioned above, the pricing problem is strongly $\mathcal{N} \mathcal{P}$-hard. Hence, if we want to use the column generation to compute a cost share, we must enlarge the set of columns. The standard way to do this in the literature is to add columns representing infeasible routes in which customers are permitted to be visited more than once. It is well known that the pricing problem then becomes a capacitated shortest path problem, which can be solved in pseudo-polynomial time by dynamic programming (see again, e.g., [4]).

If, on the other hand, we want to compute a cost share in polynomial time, we can use the cutting plane approach based on assignable inequalities. It is easy to show that all of the inequalities which are assignable for the traveling salesman game are also assignable for the vehicle routing game. Moreover, further assignable inequalities were presented in [25]. (They were called decomposable inequalities in that paper.) These include the following Generalized Large Multistar (GLM) inequalities [21,24]:

$$
\sum_{e \in \delta(R)} x_{e} \geq \frac{2}{Q} \sum_{i \in R}\left(q_{i}+\sum_{j \in V \backslash R} q_{j} x_{(i, j)}\right), \quad R \subseteq V .
$$

To make the present paper self-contained, we give a proof here:

Theorem 9 For the vehicle routing game, the GLM inequalities (Eq. 32) are assignable.

Proof It suffices to show that the (homogeneous) inequalities

$$
\sum_{e \in \delta(R)} x_{e} \geq \frac{2}{Q}\left(\sum_{i \in R}\left(q_{i} y_{i}+\sum_{j \in V \backslash R} q_{j} x_{(i, j)}\right)\right), \quad R \subseteq V
$$

are valid for $P_{I}^{x y}$. So let $(\bar{x}, \bar{y})$ be an extreme point of $P_{I}^{x y}$. Note that we can assume, without loss of generality, that there is no vertex $j \in V \backslash R$ with $\sum_{i \in R} \bar{x}_{(i, j)}=2$, since, if there was, we could decrease the slack (or increase the violation) of the inequality (Eq. 33) by inserting $j$ into $R$.

Now, it is clear that the set of vehicles entering and leaving $R$ has to visit not only the vertices in $R$ with $\bar{y}_{i}=1$, but also any vertex $j \in V \backslash R$ such that $\bar{x}_{(i, j)}=1$ for some $i \in R$. Under the above assumption, the total demand of these vertices is equal to:

$$
\sum_{i \in R}\left(q_{i} \bar{y}_{i}+\sum_{j \in V \backslash R} q_{j} \bar{x}_{(i, j)}\right)
$$


and therefore at least

$$
\frac{1}{Q} \sum_{i \in R}\left(q_{i} \bar{y}_{i}+\sum_{j \in V \backslash R} q_{j} \bar{x}_{(i, j)}\right)
$$

vehicles must enter and leave $R$. The validity of Eq. 33 follows.

Theorem 10 For the vehicle routing game, a cost share exists whose value is at least the value of the LP relaxation of the vehicle routing problem with non-negativity constraints, degree equations for $i \in V$, subtour elimination constraints, weakened clique tree inequalities, and GLM inequalities.

As the GLM inequalities (Eq. 32) can be separated efficiently by max-flow techniques [21,24], we have:

Corollary 9 For the vehicle routing game, we can find in polynomial time a cost share whose value is at least equal to the value of the LP relaxation of the vehicle routing problem with non-negativity constraints, degree equations for $i \in V$, subtour elimination constraints, weakened blossom inequalities, and GLM inequalities.

Other assignable inequalities discussed in [25] include the Knapsack Large Multistar (KLM) inequalities [24], which are a generalization of the GLM inequalities, and the so-called hypotour-like inequalities. While the complexity of the separation problem for the KLM inequalities is unknown, a generalization of the hypotour-like inequalities can be separated efficiently [25].

Finally, we briefly consider the combination of both row and column generation. As shown in [25], the GLM inequalities are already implied by the set partitioning formulation, even when non-elementary routes are permitted. Thus, there is no advantage to including a separation algorithm for the GLM inequalities in the cut-and-price approach. However, there usually is a benefit to be obtained by including a separation algorithm for the SECs. The resulting lower bound on $c(V)$ is typically fairly close to $c(V)$ itself [11].

\section{Conclusions}

We have defined a very general class of TU games, the IM games, and given a general framework for computing good cost shares. This framework allows one to apply two highly successful optimization techniques - cutting planes and column generationto a broad class of combinatorial optimization games. We have also analysed some particular IM games in detail. We remark that the same methods can be applied also to the Mixed-Integer variant of IM games, in which only a subset of the components of $x$ are required to be integer in Eq. 4 .

There are several interesting topics for future research. In the first place, one could search for classes of assignable inequalities for polyhedra associated with particular IM games of interest. Secondly, one could try to analyze the worst-case ratios between $c(V)$ (the optimal value of the underlying combinatorial optimization problem), the 
value of the optimal cost share, and the value of the cost shares that can be computed efficiently by the methods described in this paper. Thirdly, a practical implementation of these methods would be of interest, possibly for IM games in general. Finally, one could attempt to extend our approach to the case of non-TU games.

Acknowledgments We are grateful to Michel Goemans for illuminating discussions on the subject, and to two anonymous referees for their helpful comments.

\section{References}

1. Bauer, P.: The circuit polytope: facets. Math. Oper. Res. 22, 110-145 (1997)

2. Bläser, M., Shankar Ram, L.: Approximately fair cost allocation in metric traveling salesman games. Theory Comput. Syst. 43, 19-37 (2008)

3. Bondareva, O.N.: Some applications of linear programming to cooperative games. Problemy Kibernetiki. 10, 119-139 (1963)

4. Bramel, J., Simchi-Levi, D. : Set-covering-based algorithms for the capacitated VRP. In: Toth, P., Vigo, D. (eds.) The Vehicle Routing Problem, SIAM Monographs on Discrete Mathematics and Applications SIAM, Philadelphia (2002)

5. Dantzig, G.B., Wolfe, P.: Decomposition principle for linear programs. Oper. Res. 8, 101-111 (1960)

6. de Aragaõ, M.P., Uchoa, E.: Integer program reformulation for robust branch-and-cut-and-price. In: Wosley, L. (ed.) Annals of Mathematical Programming in Rio, pp. 56-61. Búzios, Brazil (2003)

7. Deng, X., Ibaraki, T., Nagamochi, H.: Algorithmic aspects of the core of combinatorial optimization games. Math. Oper. Res. 24, 751-766 (1999)

8. Edmonds, J.: Maximum matching and a polyhedron with 0,1-vertices. J. Res. Natl. Bur. Stand. 69, 125$130(1965)$

9. Faigle, U., Fekete, S.P., Hochstättler, W., Kern, W.: On approximately fair cost allocation for the Euclidean traveling salesman problem. OR Spektrum 20, 29-37 (1998)

10. Fekete, S.P., Pulleyblank, W.R.: A note on the traveling preacher problem. Report No. 98.331, Angewandte Mathematik und Informatik, Universität zu Köln (1998)

11. Fukasawa, R., Lysgaard, J., de Aragão, M.P., Reis, M., Uchoa, E., Werneck, R.F.: Robust branch-andcut-and-price for the capacitated vehicle routing problem. In Nemhauser, G., Bienstock, D. (eds.) Integer Programming and Combinatorial Optimization 10, pp. 1-15. Lecture Notes in Computer Science 3064, Berlin, Springer (2004)

12. Gillies, D.B: Solutions to general non-zero-sum games. In: Tucker, A.W., Luce, R.D. (eds.) Contributions to the Theory of Games, vol. 4, pp. 47-85. Princeton University Press, Princeton (1959)

13. Goemans, M.X.: Private communication (2004)

14. Goemans, M.X., Skutella, M.: Cooperative facility location games. J. Algorithms 50, 194-214 (2004)

15. Göthe-Lundgren, M., Jörnsten, K., Värbrand, P.: On the nucleolus of the basic vehicle routing game. Math. Program. 72, 83-100 (1996)

16. Granot, D., Huberman, G.: Minimum cost spanning tree games. Math. Program. 21, 1-18 (1981)

17. Grötschel, M., Lovász, L., Schrijver, A.: Geometric Algorithms and Combinatorial Optimization. Springer, Berlin (1988)

18. Grötschel, M., Padberg, M.W.: On the symmetric travelling salesman problem I: inequalities. Math. Program. 16, 265-280 (1979)

19. Grötschel, M., Padberg, M.W.: Polyhedral theory. In: Lawler, E.L. et al. (ed.) The Traveling Salesman Problem: A Guided Tour of Combinatorial Optimization Wiley, Chichester (1985)

20. Grötschel, M., Pulleyblank, W.: Clique tree inequalities and the symmetric travelling salesman problem. Math. Oper. Res. 11, 537-569 (1986)

21. Gouveia, L.: A result on projection for the vehicle routing problem. Eur. J. Opl. Res. 85, 610-684 (1995)

22. Houck, D.J., Picard, J.-C., Queyranne, M., Vemuganti, R.R.: The travelling salesman problem as a constrained shortest path problem: theory and computational experience. Opsearch 17, 93-109 (1980)

23. Jain, K., Mahdian, M.: Cost sharing. In: Nisan, N., Roughgarden, T., Tardos, E., Vazirani, V. Algorithmic Game Theory, Cambridge University Press, Cambridge (2007)

24. Letchford, A.N., Eglese, R.W., Lysgaard, J.: Multistars, partial multistars and the capacitated vehicle routing problem. Math. Program. 94, 21-40 (2002) 
25. Letchford, A.N., Salazar Gonzalez, J.J.: Projection results for vehicle routing. Math. Program. 105, 251-274 (2006)

26. Naddef, D.: Polyhedral theory and branch-and-cut algorithms for the symmetric TSP. In: Gutin, G., Punnen, A. (eds.) The Traveling Salesman Problem and Its Variations, Kluwer, Dordrecht (2001)

27. Naddef, D., Rinaldi, G.: The symmetric traveling salesman polytope and its graphical relaxation: composition of valid inequalities. Math. Program. 51, 359-400 (1991)

28. von Neumann, J., Morgenstern, O.: Theory of Games and Economic Behaviour. Princeton University Press, Princeton (1947)

29. Okamoto, Y.: Traveling salesman games with the Monge property. Discr. Appl. Math. 138, 349369 (2004)

30. Padberg, M.W., Rao, M.R.: Odd minimum cut-sets and $b$-matchings. Math. Oper. Res. 7, 67-80 (1982)

31. Pal, M., Tardos, E.: Group strategy proof mechanisms via primal-dual algorithms. In: Proceedings of the 44th annual IEEE symposium on foundations of computer science (FOCS 2003), pp. 584-593 (2003)

32. Peleg, B., Sudholter, P.: Introduction to the Theory of Cooperative Games. Kluwer, Dordrecht (2003)

33. Potters, J.A.M., Curiel, I.J., Tijs, S.H.: Traveling salesman games. Math. Program. 53, 199-211 (1992)

34. Seymour, P.D.: Sums of circuits. In: Bondy, J.A., Murty, U.S.R. (eds.) Graph Theory and Related Topics, pp. 341-355. Academic Press, London (1979)

35. Shapley, L.S.: On balanced sets and cores. Nav. Res. Log. Quart. 14, 453-460 (1967)

36. Shapley, L.S., Shubik, M.: The assignment game I: the core. Int. J. Game Theory 1, 111-130 (1972)

37. Shubik, M.: Game theory models and methods in political economy. In Arrow, K.J., Intriligator, M.D. (eds.) Handbook of Mathematical Economics, vol. I. North-Holland, pp. 285-330 (1981)

38. Tamir, A.: On the core of a traveling salesman cost allocation game. Oper. Res. Lett. 8, 31-34 (1988) 